\title{
Far Field Extrapolation from Near Field Interactions and Shielding Influence Investigations Based on a FE-PEEC Coupling Method
}

\author{
Edith Clavel $^{1, *}$, Thanh-Son Tran ${ }^{2}$, Jérémie Aimé ${ }^{3}$, Gérard Meunier ${ }^{1}$ and James Roudet ${ }^{1}$ \\ 1 Grenoble Electrical Engineering Laboratory BP46, Domaine Universitaire, Saint Martin d'Hères \\ 38402, France; E-Mails: gerard.meunier@g2elab.grenoble-inp.fr (G.M.); \\ james.roudet@g2elab.grenoble-inp.fr (J.R.) \\ 2 Electric Power System Department, Electric Power University, 235 Hoang Quoc Viet, Hanoi \\ 10000, Vietnam; E-Mail: tranthanhson@live.com \\ 3 Cedrat, 15, Chemin de Malacher-Inovallée, Meylan Cedex 38246, France; \\ E-Mail: jeremie.aime@cedrat.com \\ * Author to whom correspondence should be addressed; \\ E-Mail: edith.clavel@g2elab.grenoble-inp.fr; Tel.: +33-4-7682-6287; Fax: +33-4-7682-6300.
}

Received: 8 October 2012; in revised form: 19 January 2013 / Accepted: 5 February 2013 /

Published: 8 March 2013

\begin{abstract}
Regarding standards, it is well established that common mode currents are the main source of far field emitted by variable frequency drive (VFD)-cable-motor associations. These currents are generated by the combination of floating potentials with stray capacitances between these floating potential tracks and the mechanical parts connected to the earth (the heatsink or cables are usual examples). Nowadays, due to frequency and power increases, the systematic compliance to EMC (ElectroMagnetic Compatibility) becomes increasingly difficult and costly for industrials. As a consequence, there is a well-identified need to investigate practical and low cost solutions to reduce the radiated fields of VFD-cable-motor associations. A well-adapted solution is the shielding of wound components well known as the major source of near magnetic field. However, this solution is not convenient, it is expensive and may not be efficient regarding far field reduction. Optimizing the components placement could be a better and cheaper solution. As a consequence, dedicated tools have to be developed to efficiently investigate not easy comprehendible phenomena and finally to control EMC disturbances using component placement, layout geometry, shielding design if needed. However, none of the modeling
\end{abstract}


methods usually used in industry complies with large frequency range and far field models including magnetic materials, multilayer $\mathrm{PCBs}$, and shielding. The contribution of this paper is to show that alternatives regarding modeling solutions exist and can be used to get in-deep analysis of such complex structures. It is shown in this paper that near field investigations can give information on far field behavior. It is illustrated by an investigation of near field interactions and shielding influence using a FE-PEEC hybrid method. The test case combining a common mode filter with the floating potentials tracks of an inverter is based on an industrial and commercialized VFD. The near field interactions between the common mode inductance and the tracks with floating potentials are revealed. Then, the influence of the common mode inductance shielding is analyzed.

Keywords: electromagnetic compatibility; electromagnetic coupling; modeling; power conversion

\section{Introduction}

Many studies have been carried out on EMC (ElectroMagnetic Compatibility) modeling of power electronic devices. Indeed, this field of research is closely linked to industrial constraints and needs, inherited from standards. Considering EMC purpose, developing reliable models in the early phase of the design of power electronics devices is a suitable solution. Several Computer Aided Design (CAD) tools have been developed as such [1-4]. Unfortunately, model computing far field models of industrial products with complex geometries are still very difficult to implement. In the radiated EMC domain, the usual modeling methods are necessarily limited by the considered frequency range and/or by the geometry complexity, as well as the medium heterogeneity. Consequently, many works are focused on modeling methods that are able to take all these parameters into account [5-7]. Engineers thus need new solutions to improve their modeling approach during the design stage. In other words, modeling environments must be robust, and implemented in CAD tools with easy-to-use interfaces.

These CAD tools must give quite complete models with parametric descriptions, so that industrial solutions can be improved. That is why layout techniques with a placement of components limiting EMC disturbances have been investigated [8-12]. These techniques must lead to practical solutions for customized devices. These solutions are very interesting because they improve industrial products without increasing the Joule losses and are effective for a high frequency range which is not the case of the usual passive solutions (such as the addition of a filter). The developed models must be accurate and flexible enough to efficiently improve power electronic structures thus reducing the number of prototypes.

To simplify the problem a near field analysis is developed in this paper. Shielding, layout complexity and component placement are taken into account. Moreover, from this analysis the behavior in far field can be deduced. The modeling method is presented in [7]. It is based on the coupling between the Finite Elements and PEEC methods, implemented in commercialized software [13]. The method is applied to a test case, which is extracted from a commercialized VFD and composed of the common mode filter and the output tracks of the inverter. The near field 
interactions between the floating potential tracks where common mode currents are generated, and the common mode inductance which is a source of magnetic field are evaluated. A study of the shielding is also carried out. It is shown that this very common solution is not always the most suitable one to adopt and better performances can be reached with cheaper solutions.

In a first part, the mechanisms linked to the generation of EMC perturbations in power converters are presented in order to explain how near field interactions can impact far field levels. In a second part, the usual modeling methods are presented and their limitations are highlighted. Regarding the complexity of required models these classical approaches need to be improved. A FE-PEEC coupling solution is used and presented [7]. Finally, an application of the proposed modeling approach is shown. The complexity of the test case is highlighted and the influence of component placement and shielding on the far field is deduced from the near field analysis.

\section{EMC Study of Static Converters}

The increase of power and frequency amplifies the disturbance level. Moreover, for marketing considerations, an increase of performance must not be threatened by the level of pollution of new structures. The means of disturbance reduction depend on the frequency range, which extends from some Hertz to a few Gigahertzes. Electromagnetic disturbances can be separated into two bands: Below $30 \mathrm{MHz}$, the conducted ones are preponderant, beyond is the domain of radiated disturbances. In our case, the studied power electronics structure is a VFD. Multilayer printed circuits are often used because of thermal constraints. As a consequence, a full modeling of the geometries can be complex. Moreover, depending on the applications and the power range, the switching frequency is usually comprised between $10 \mathrm{kHz}$ and $100 \mathrm{kHz}$. Conducted band studies show that the spectrum shapes are closely linked to the driver configurations. Indeed, EMC perturbations of a static converter are due to the differential and common mode currents $[10,14]$. The latter are generated by the floating potential tracks with their stray capacitances referenced to the ground planes during the switching stages (Voltage driven mechanism) and by the variation of ground plane voltage due to the stray inductances (Current driven mechanism).

Considering the static converter as an antenna, the semi-conductor component is the source and the cabling is the path. While switching, a semi-conductor component generates a high $\mathrm{d} v / \mathrm{d} t$, which is transformed into a common mode current via the parasitic capacitance between the conducting tracks and the ground planes. The current and voltage driven mechanisms are combined in VFD-cable-motor associations. Moreover, this study is focused more particularly on voltage driven mechanism because ground planes are not inductive enough and capacitive behavior is the dominant part of stray impedance in VFD. In addition, interactions with the layout are potentially responsible for eddy currents generation. These eddy currents, which are generated by near field couplings can increase the common mode generation of current. Finally, the common mode current loops generates magnetic field (Biot and Savart's law) and $\mathrm{d} v / \mathrm{d} t$ generates electric field-radiated by these tracks. This is illustrated on Figure 1.

These phenomenons can be measured. As an example, Figure 2 shows the field along (Oy) of a boost converter at 3 meters and its common mode voltage (Voltage drop between the floating potential and the ground plane). Only the field emitted by the converter is measured, whereas the influence of cables, power supply, load and driver is neglected since they are placed behind copper planes. The two curves resonances are clearly correlated. This is also explained by the fact that radiated field of wound 
components quickly decreases regarding the distance from the source [15]. Therefore, it can be said that the far field is mainly correlated to the common mode. In conclusion, to reduce or control the far field emissions, it is necessary to control the common mode generation [10]. It is thus essential to preserve the floating potential tracks from electromagnetic disturbances.

A useful solution is to shield the main near magnetic sources, here the common mode inductance. However, this drastically increases the weight and cost of the final product. In order to quantify the impact of the couplings and shielding on floating potentials, models need to be developed.

Figure 1. Voltage driven mechanism of common mode current generation.

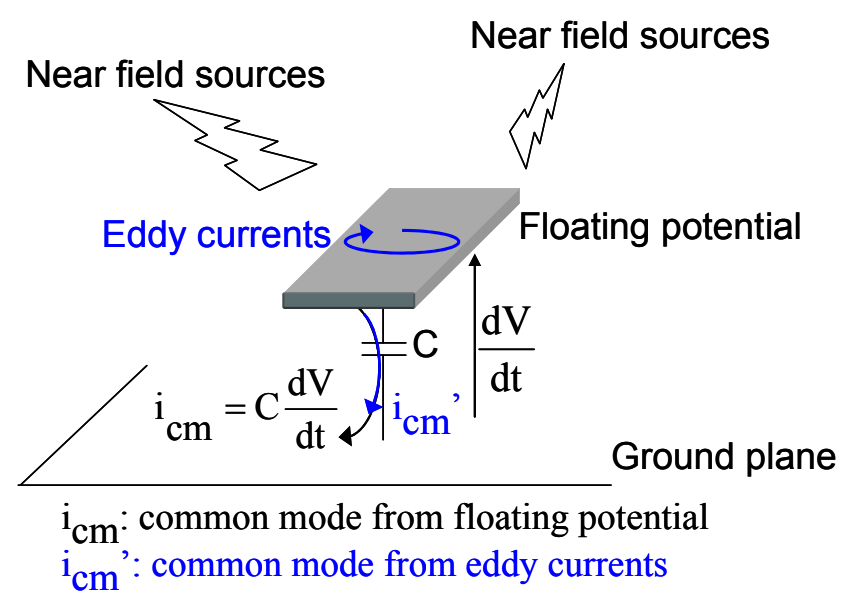

Figure 2. Correlation between far field and common mode.
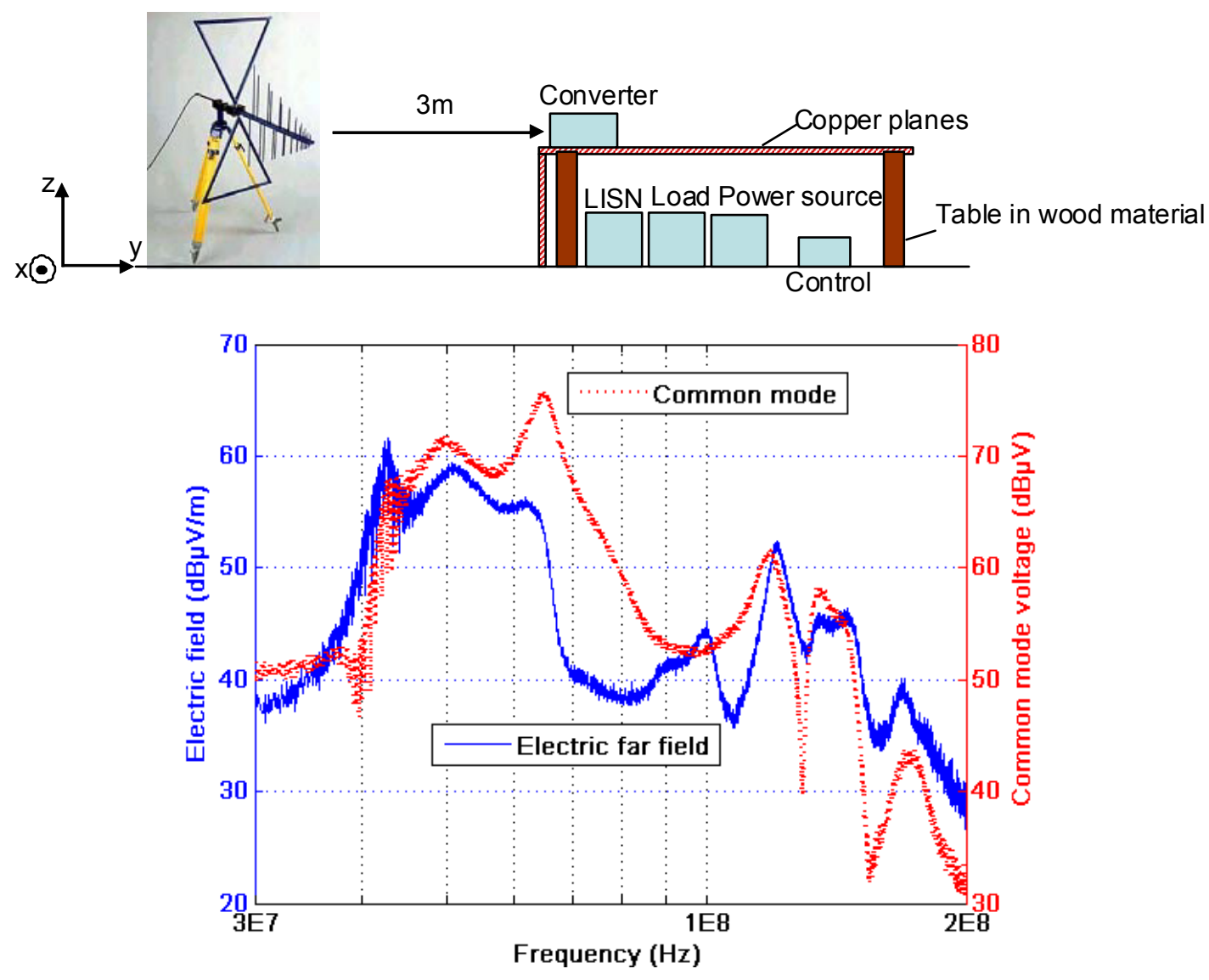


\section{State of the Art on Modeling Methods}

\subsection{Usual Methods}

Electrical equivalent models are necessary to take into account EMC constraints during the design process. According to the studied component, the modeling method that will be used to evaluate EMC performances will be different, depending on its nature, the required accuracy and the level of the model. Models have to consider heterogeneity of media (Magnetic components, copper tracks, substrates such as FR4 for example and air) and multilayer topologies.

On the one hand, Method of Moments (MoM) and method of Partial Element Equivalent Circuits (PEEC) are useful because they are not expensive in computational time. Indeed, they do not require meshing the whole considered space [16,17]. On the other hand, the Finite Element Method (FEM) takes into account inhomogeneous environments and magnetic materials [18]. Unfortunately, a fine meshing is needed to obtain accurate results. Moreover, all space is considered and computational time can become too costly for complex geometries. An alternative could be the development of coupling methods. As a consequence, works are carried out on the development of modeling methods making it possible to take into account these elements [5-7]. Moreover, due to the considered geometries, dedicated tools with adapted interfaces are really needed.

\subsection{Hybrid Method}

A complex system such as motor drivers is made of many thin conductors and ferromagnetic materials. Modeling them using FEM requires a lot of meshes, which becomes too problematic to find a solution. For power electronics applications, this step has to answer two very strict conditions:

- Close to the conductors, variations of magnetic field is very important [19], so to take this aspect into account, the meshing must be dense;

- Geometry of conductors is very hard to mesh with a good quality since it can be constituted by very this planes like DBC (Direct Copper Board).

Indeed, a good quality meshing requires two meshes into the skin depth for the skin effect to take into account.

On the other hand, because of three dimensional ferromagnetic materials, it is very complicated to solve this problem by PEEC method. Hence a hybrid method dealing with this type of problem was developed [7]. The idea of the hybrid method is to benefit from the main advantage both of FEM and PEEC method:

- PEEC method easily takes into account interactions between complex 3D massive conductors;

- FEM takes the interaction between conductors and ferromagnetic materials into account.

One of the advantages of PEEC method is a simple meshing of conductors since assumptions on their current density can be applied. Skin and induced effects can be easily modeled. Each element of FEM meshing is considered as an inductor with a uniform current density. In other words, a conductor is represented by a set of inductors using this hybrid method. Air around conductors and magnetic regions are still meshed using finite elements.

A general problem with inductors and ferromagnetic material is shown in Figure 3. 
Figure 3. General description of FEM-PEEC coupled method.

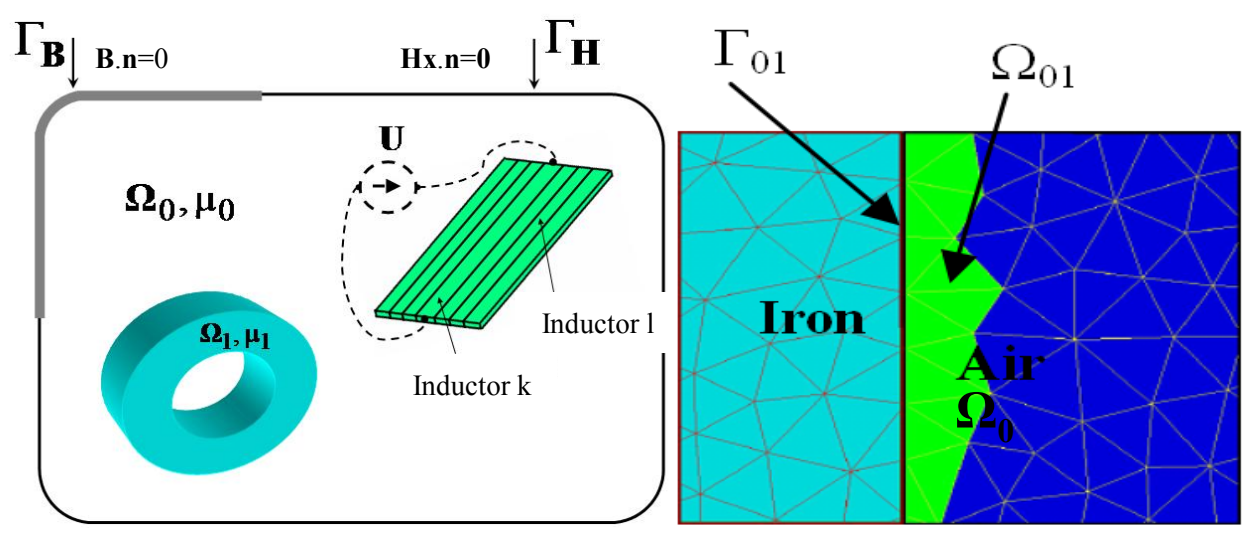

To adapt FEM to the coupling, the magnetic scalar potential formulation is used. With this formulation, the magnetic field is calculated by a total scalar potential $\phi[20]$ and a reduced field $T_{0}$ due to all inductors as Equation (1).

$$
\begin{aligned}
& H=T_{0}-\operatorname{grad} \phi \text { in } \Omega_{0} \\
& H=-\operatorname{grad} \phi \text { in } \Omega_{1}
\end{aligned}
$$

$\Omega_{0}$ is the region with air including the conductors. $\Omega_{1}$ is the ferromagnetic region. $\boldsymbol{T}_{0}$ can be expressed with $\boldsymbol{t}_{0 \mathrm{k}}$ representing the source of $1 \mathrm{~A}$ in the inductor $\mathrm{k}$ and its current $I_{\mathrm{k}}(2)$.

$$
T_{0}=\sum_{k=1}^{m} t_{0 \mathrm{k}} \mathrm{I}_{\mathrm{k}}
$$

Because curl $H=j$ in $\Omega_{0}$ where $j$ is local current density, so curl $T_{0}=j$.

If $j_{0 \mathrm{k}}$ is the current density of inductor $\mathrm{k}$ fed by a current of $1 \mathrm{~A}, t_{0 \mathrm{k}}$ has to satisfy (3) and (4).

$$
\begin{aligned}
& \operatorname{curl} t_{0 \mathrm{k}}=j_{0 \mathrm{k}} \text { in } \Omega_{0} \\
& t_{0 \mathrm{k}} \times \mathrm{n}=0 \text { on } \Gamma_{01}
\end{aligned}
$$

Equation (4) is imposed because of $H_{\mathrm{t}}$ continuity on the interface $\Gamma_{01} . t_{0 \mathrm{k}}$ is expressed by (5).

$$
t_{0 \mathrm{k}}=h_{0 \mathrm{k}}-\operatorname{grad} \delta \phi_{\mathrm{k}}
$$

In Equation (5), $h_{0 \mathrm{k}}$ is the Biot and Savart magnetic field and $\delta \phi_{\mathrm{k}}$ is the jump between scalar and reduced potentials [12] satisfying (4).

Note that this formulation does not require any meshing of the inductors but the source field $\mathrm{t}_{0 \mathrm{k}}$ has to be estimated before the finite element resolution. The weak form of $\mathrm{T}_{0}-\phi$ formulation leads to the finite element matrix system (6).

$$
[\mathrm{A}]\{\phi\}=-[\mathrm{C}]\{\mathrm{I}\}
$$

In Equation (6), terms of matrices $\mathrm{A}$ and $\mathrm{C}$ are defined by (7). 


$$
\begin{aligned}
& \mathrm{A}_{\mathrm{ij}}=\int_{\Omega} \mu \operatorname{grad} \alpha_{\mathrm{i}} \cdot \operatorname{grad} \alpha_{\mathrm{j}} \mathrm{d} \Omega \\
& \mathrm{C}_{\mathrm{ik}}=-\int_{\Omega_{0}} \mu_{0} \operatorname{grad} \alpha_{\mathrm{i}} \cdot t_{0 \mathrm{k}} \mathrm{d} \Omega
\end{aligned}
$$

Where $\alpha_{i}$ and $\alpha_{j}$ are the nodal finite element interpolation functions approximating $\phi$. If the inductor $\mathrm{k}$ is fed by a voltage source $U_{\mathrm{k}}$, the current $I_{\mathrm{k}}$ is unknown and a circuit relation must be added [21] (8).

$$
\mathrm{U}_{\mathrm{k}}=\mathrm{R}_{\mathrm{k}} \mathrm{I}_{\mathrm{k}}+\mathrm{j} \omega \int_{\Omega_{0}} t_{0 k} \cdot B \mathrm{~d} \Omega
$$

The magnetic induction in $\Omega_{0}$ (air) can be written using (9).

$$
B=\mu_{0} H=\mu_{0}\left[\sum_{k=1}^{m} t_{0 \mathrm{k}} \mathrm{I}_{\mathrm{k}}-\operatorname{grad} \phi\right]
$$

Combining (6) and (8) gives (10).

$$
\left[\begin{array}{cc}
A & C \\
C^{t} & D+R
\end{array}\right]\left\{\begin{array}{l}
\phi \\
I
\end{array}\right\}=\left\{\begin{array}{c}
0 \\
\frac{U}{j \omega}
\end{array}\right\}
$$

In equation (10), terms of matrices $\mathrm{D}$ and $\mathrm{R}$ are defined by (11).

$$
\mathrm{D}_{\mathrm{kl}}=\int_{\Omega_{0}} \mu_{0} \mathbf{t}_{0 \mathrm{k}} \cdot \mathbf{t}_{01} \mathrm{~d} \Omega \quad \mathrm{R}_{\mathrm{kk}}=\left[\frac{\mathrm{R}_{\mathrm{k}}}{\mathrm{j} \omega}\right]
$$

Computations of $\mathrm{D}_{\mathrm{kl}}$ require a fine meshing around inductors because the variation of $t_{0 \mathrm{k}}$ and $t_{01}$ is very strong around them. To avoid this problem, a coupling of this formulation with PEEC method was proposed. $D_{k l}$ is calculated by the mutual inductance $M_{k l}$ that can be exactly determined by PEEC method. Substituting (5) into (11) gives (12).

$$
\mathrm{D}_{\mathrm{kl}}=\int_{\Omega_{0}} \mu_{0} \mathbf{h}_{0 \mathrm{k}} \cdot \mathbf{t}_{01} \mathrm{~d} \Omega-\int_{\Omega_{0}} \mu_{0} \operatorname{grad} \delta \phi_{\mathrm{k}} \cdot \mathbf{t}_{01} \mathrm{~d} \Omega
$$

The first term of right part of (12) represents the mutual inductance in vacuum between inductor $k$ and $l[20]$. The second term can be transformed into surface integral by applying divergence theorem (13).

$$
\mathrm{D}_{\mathrm{kl}}=\mathrm{m}_{\mathrm{kl}}-\int_{\Gamma_{01}} \mu_{0} \delta \phi_{\mathrm{k}} h_{01} \cdot n \mathrm{~d} \Gamma+\int_{\Omega_{01}} \mu_{0} \operatorname{grad} \delta \phi_{\mathrm{k}} \cdot \operatorname{grad} \delta \phi \mathrm{d} \Omega
$$

Note that the terms $C_{i k}$ in (7) can be transformed as follow (14).

$$
\mathrm{C}_{\mathrm{ik}}=-\int_{\Gamma_{01}} \mu_{0} \alpha_{\mathrm{i}} h_{0 \mathrm{k}} \cdot n \mathrm{~d} \Gamma+\int_{\Omega_{01}} \mu_{0} \operatorname{grad} \alpha_{\mathrm{i}} \cdot \operatorname{grad} \delta \phi_{\mathrm{k}} \mathrm{d} \Omega
$$

This avoids estimating $t_{0}$ in entire domain. $t_{0}$ is computed only on the interface $\Gamma_{01}$ and on a layer of elements $\left(\Omega_{01}\right.$, Figure 3$)$. In conclusion, the proposed hybrid method avoids pre-calculating of $t_{0}$ in entire domain and relaxes the meshing around conductors. This was validated and gives stable results [7,19]. A case of study is defined in order to analyze this phenomenon using this original modeling method. 


\section{Application and Results}

\subsection{Studied Structure}

The chosen case of study is a simplified structure extracted from the geometry of an industrial variable speed drive. It is composed of a three-phase common mode filter with the floating potential tracks (U, V, W) of an inverter (Figures 4 and 5).

Figure 4. Description of the studied layout.

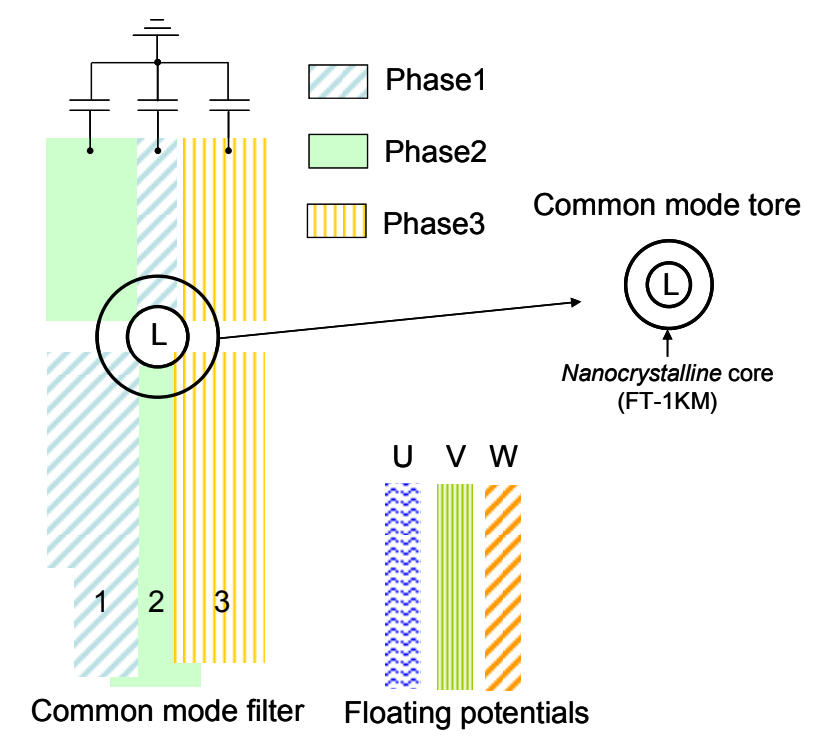

Figure 5. Electrical topology to model interactions.

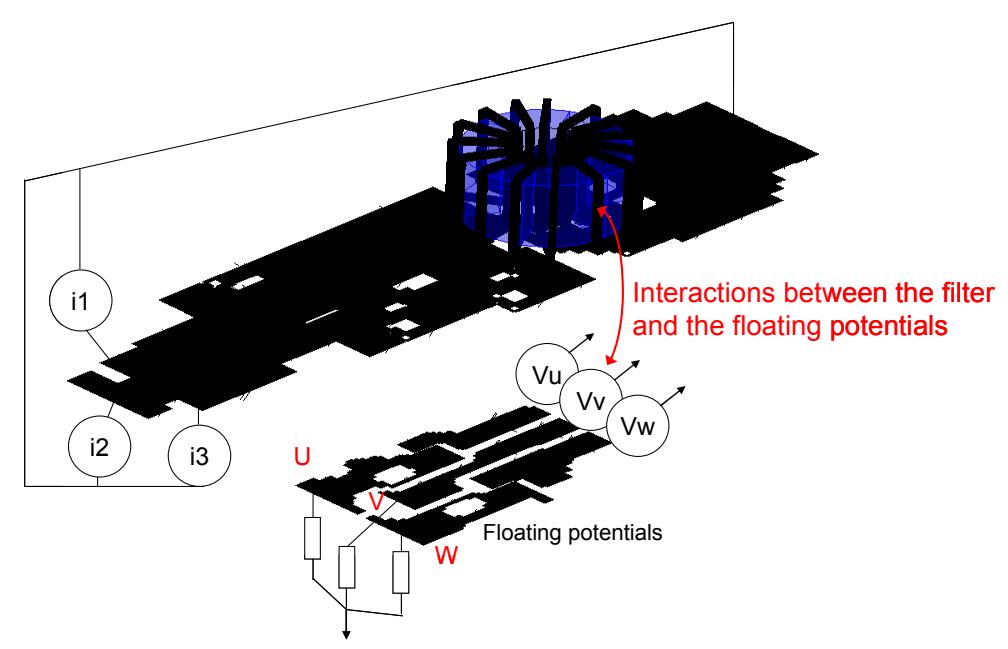

The toroidal core inductance material is the FT-1KM nanocrystalline, manufactured by Hitachi.

This material has a higher permeability than ferrite; it is first-rated for common mode filtering applications. The initial permeability $\mu \mathrm{r}$ is equal to 16,000 at $20^{\circ} \mathrm{C}$ and for a frequency equal to $100 \mathrm{kHz}$. Each winding is composed of five turns. The theoretical value of the inductance is $1.2 \mathrm{mH}$. Due to the winding turns, the inductance is the main source in near field. Moreover, because of mutual couplings with the other parts of the structure, additional perturbations can occur. 
Regarding the PCB, it is composed of two layers. Phase 1 and 2 are crossed. Phase 3 and the floating potentials $\mathrm{U}, \mathrm{V}$ and $\mathrm{W}$ are routed on the top layer. The copper width is equal to $70 \mu \mathrm{m}$. The resolution frequency is chosen equal to the switching frequency $(10.6 \mathrm{kHz})$.

Using the hybrid method that is described in the previous section, the cabling is modeled using PEEC approach and the magnetic material of the inductance using the FE method.

The aim of the study is to evaluate the interactions between the filter and the floating potentials tracks which are the most influent part regarding common mode current generation $(\mathrm{ic}=\mathrm{C} . \mathrm{d} V / \mathrm{d} t)$ and by this way, far field emissions (Figure 5).

\subsection{Results}

The currents of floating potentials tracks are computed with and without the common mode filter. Hence the influence of the couplings between the floating potential tracks $\mathrm{U}, \mathrm{V}, \mathrm{W}$, and the common mode filter can be analyzed. In addition, Table 1 shows that the couplings are not negligible between the common mode filter and the floating potentials tracks with unbalanced currents of higher values.

Table 1. Coupling and shielding influences on the currents of floating potentials tracks.

\begin{tabular}{cccc}
\hline \multirow{2}{*}{ Currents without filter (A) } & $\mathrm{U}$ & $\mathrm{V}$ & $\mathrm{W}$ \\
\cline { 2 - 4 } & 328.628 & 373.487 & 403.316 \\
\hline Currents with filter (A) & $\mathrm{U}$ & $\mathrm{V}$ & $\mathrm{W}$ \\
\cline { 2 - 4 } Without shielding (A) & 465.60 & 527.09 & 570.53 \\
With shielding (A) & 465.59 & 527.04 & 570.48 \\
\hline
\end{tabular}

Usually, for industrial devices, a well adapted solution is the shielding of wound components well known as the major source of near magnetic. Using the modeling process, a shielding has been easily added on the common mode inductance to evaluate its influence on the currents (Figure 6). It has been described in the FE part of the modeling method.

Figure 6. Common mode inductance shielding.

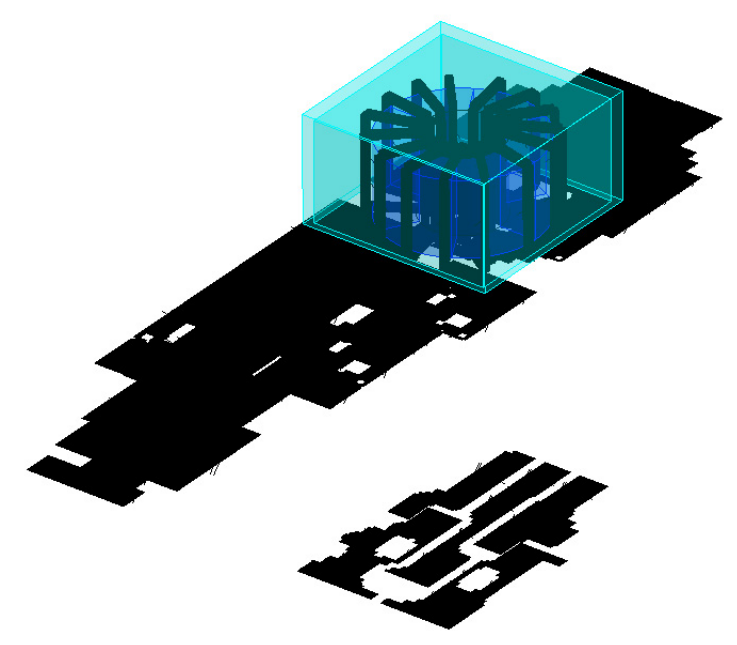

Since the shielding is made of thin plates of conducting material, the classical FE description is not the best way since the low thickness can lead to numerical errors during the solving stage. So an 
adapted meshing strategy with specific type of mesh has been applied. Its use and the associated formulations are detailed in [22,23].

As shown in Table 1, the shielding does not reduce the eddy currents. Its influence is localized around the inductance (Figure 7) and its efficiency decreasing is too fast to reach $\mathrm{U}, \mathrm{V}$, and $\mathrm{W}$ (Figure 8). In this case, the common mode inductance shielding is not an efficient solution to reduce the common mode generation.

Figure 7. Shielding influence repartition.

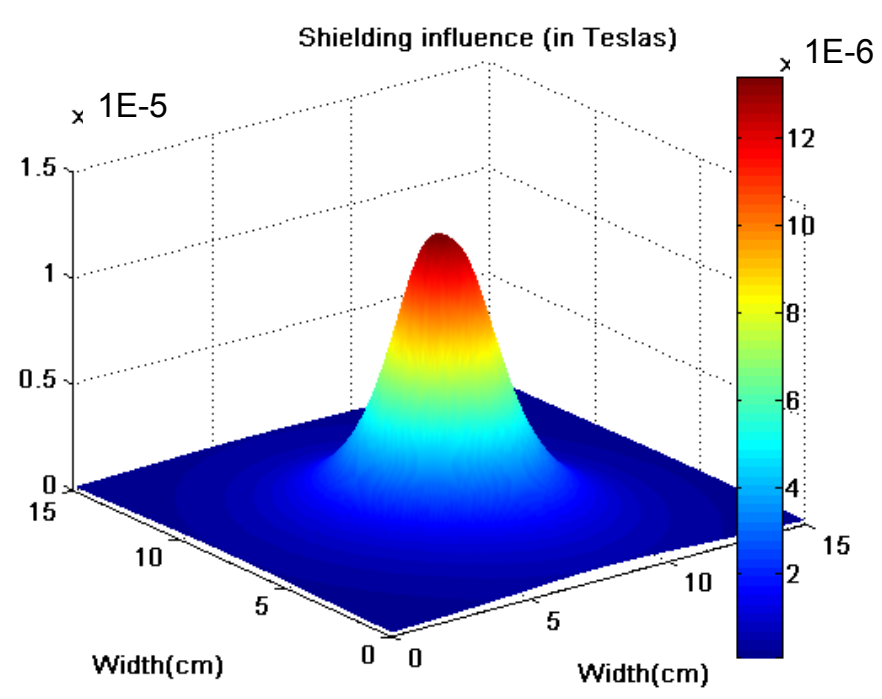

Figure 8. Shielding influence decreasing.

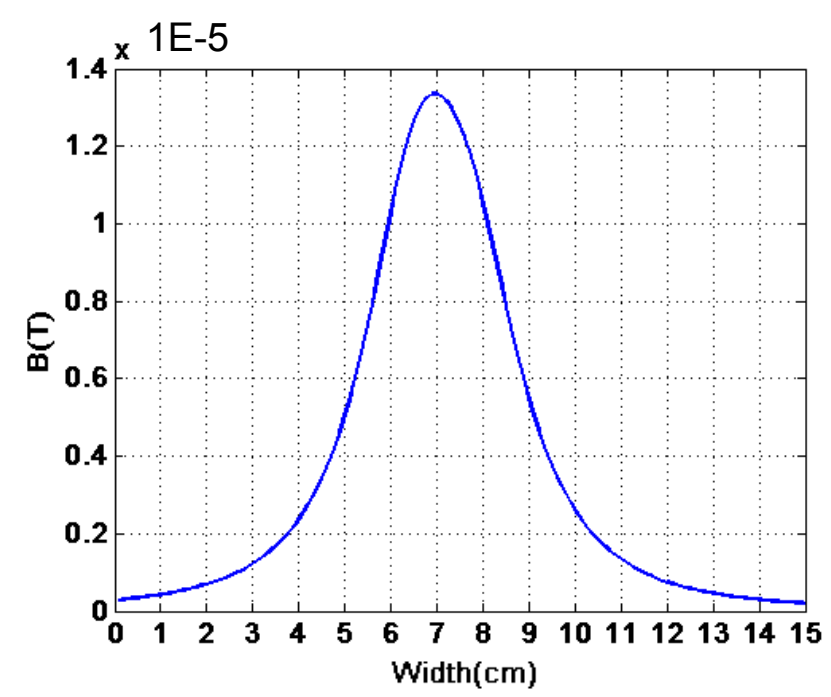

Regarding the performances of such a modeling process using this hybrid method, its solving time as well as the necessary memory space have been compared with the case of the use of only FE method. With the proposed method, they have been divided by three. Therefore, it is faster for a same problem and is able to solve more complicated structures with higher number of unknowns.

The influence of couplings has been verified by measuring the far field of variable speed drives according to the studied configuration. The emitted field is compared with a topology where the inductance is placed closer to the floating potential tracks (Figure 9). As expected, the emitted field is 
more important in this case (Figure 10). The interactions are favored by the proximity between the inductance and the floating potential tracks. The common mode currents are more influent due to the adding of induced currents in $\mathrm{U}, \mathrm{V}$ and $\mathrm{W}$.

The geometry is influent and routing process with component placement need to take into account these elements in order to reduce the possible increase of the common mode current.

Figure 9. Two positions of the common mode inductance.

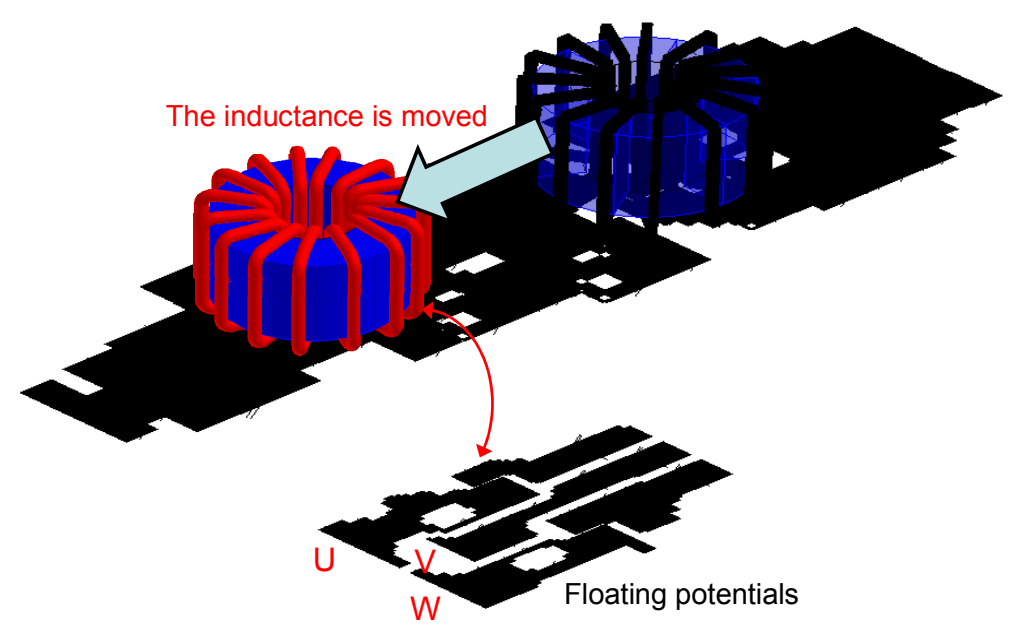

Figure 10. Far field measurement showing the influence of the interactions.

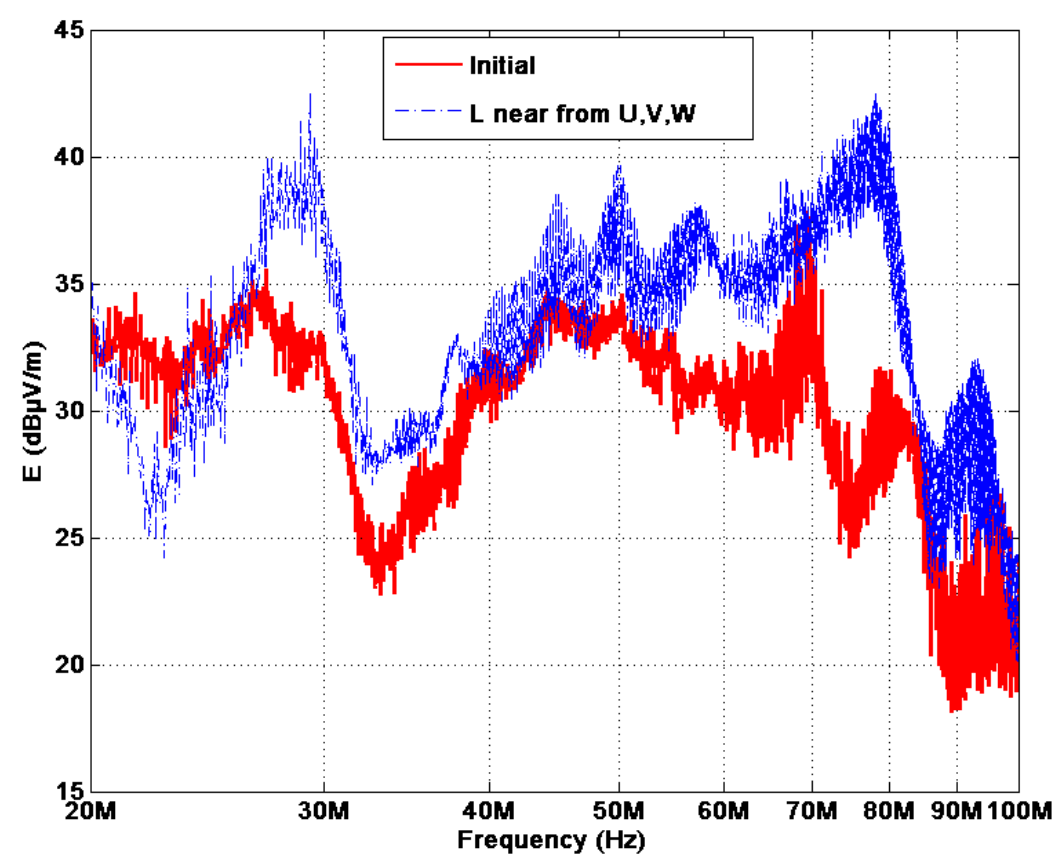

The couplings cannot be neglected in a near field computation. However, is it also the case in a far field context? As a consequence, it is now necessary to compare modeling methods in order to transpose the study in the far field domain. Works are in progress to compare FEM, PEEC and multipole expansion models in far field. For example, a static study of the inductor using FEM and multipole approach is presented on Figure 11 [15]. The magnetic field evaluation using a multipole 
approach instead of FEM is computed. This result shows the pertinence to use FEM for near field and multipole approach requiring less memory and time solving for far field studies.

Figure 11. Magnetic field decrease (modulus) vs. the distance from the source.

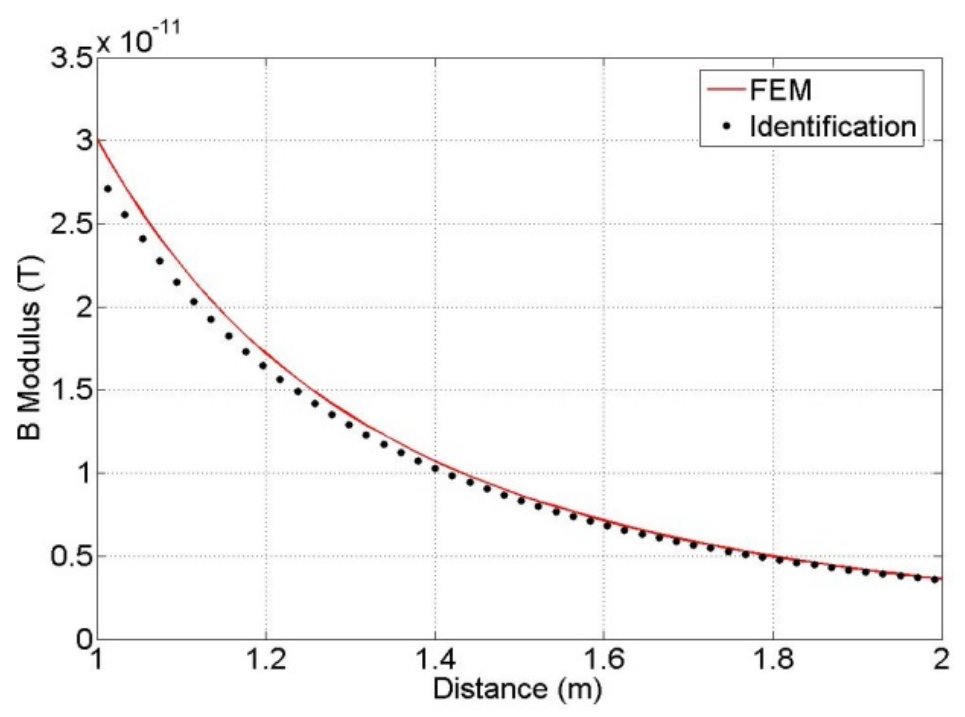

\subsection{Conclusions}

In this part, the use of the proposed hybrid method on a test case has been detailed in order to underline its advantages and the real help it can bring to designers.

Nevertheless, it can be useful for very various types of applications where both magnetic and non-magnetic components and conductors are in the same structure such as power converters.

The possible parametric description, which is not detailed here, is a real advantage when designers want to evaluate the impact of geometrical but also physical parameters on the EMC performances of the studied structure.

\section{Conclusion}

Several conclusions can be made from this analysis focusing on the far field extrapolation from near field interactions. First, these interactions cannot be neglected during the modeling stage of power electronics devices. This is demonstrated thanks to an original modeling method based on the coupling between FEM and PEEC methods. A second conclusion is that the shielding of most polluting sources is not always the most suitable solution. An adequate placement of the components, and more particularly of the floating potential tracks, is a priority. Finally, this kind of modeling approach is very useful to test some improvements on the structure, in order to satisfy the standards and to improve the EMC and EMI performances of power electronic devices. New investigations will be carried out in order to improve the approach by using multipolar expansion for the far field studies.

\section{References}

1. Wu, M.K.; Tse, C.K. Development of an integrated CAD tool for switching power supply design with EMC performance evaluation. IEEE Trans. Ind. Appl. 1998, 34, 364-373. 
2. Nagesware Rao, K.; Venkata Ramana, P. EMC analysis in PCB designs using and expert system. Int. Conf. EMI EMC 1995, 59-62.

3. Xin, W.; Pong, M.H.; Lee, C.M.; Qian, Z.M. Reduction of EMI by Electric Field Method. In Proceedings of the Fourteenth Annual Applied Power Electronics Conference and Exposition, Dallas, TX, USA, March 1999; pp. 135-138.

4. Xin, W.; Pong, M.H.; Lee, C.M.; Qian, Z.M. A Novel Approach Base on Electric Field Analysis to Reduce Crosstalk Problem on PCB. In Proceedings of the 30th Annual IEEE Power Electronics Specialists Conference, Charleston, SC, USA, March 1999; pp. 845-849.

5. Sroka, J. On the coupling of the generalized multipole technique with the finite element method. IEEE Trans. Magn. 1990, 26, 658-661.

6. Jin, J.-M.; Volakis, J.L.; Collins, J.D. A finite-element_boundary-integral method for scattering and radiation by two- and three-dimensional structures. IEEE Antennas Propag. Mag. 1991, 33, $22-32$.

7. Tran, T.-S.; Meunier, G.; Labie, P.; Le Floch, Y.; Roudet, J.; Guichon, J.-M.; Roudet, J.; Maréchal, Y. Coupling PEEC-Finite element method for solving electromagnetic problem. IEEE Trans. Magn. 2008, 44, 1330-1333.

8. Mutoh, N.; Ogata, M.; Gulez, K.; Harashima, F. New methods to suppress emi noises in motor drive systems. IEEE Trans. Ind. Electron. 2002, 49, 474-485.

9. Mutoh, N.; Nakashima, J.; Kanesaki, M. Multilayer power printed structures suitable for controlling emi noises generated in power converters. IEEE Trans. Ind. Electron. 2003, 50, 1085-1094.

10. Aimé, J.; Roudet, J.; Vollaire, C.; Baudesson, P.; Ecrabey, J. Layout Techniques for Reduction of Common Mode Current in Static Converters. In Proceedings of the 2006 IEEE Industry Applications Conference (41st IAS Annual meeting), Tampa, FL, USA, October 2006; pp. 2296-2303.

11. Aimé, J.; Clavel, E.; Roudet, J.; Meunier, G.; Loizelet, P. Electromagnetic Modelling Process to Improve Cabling of Power Electronic Structures. In Proceedings of the ISEF 2009 XIV International Symposium on Electromagnetic Fields in Mechatronics, Electrical and Electronic Engineering, Arras, France, 10-12 September 2009 [CD-ROM]; Artois Presse Université: Arras Cedex, France, 2009.

12. Yutaka, I.; Tadao, K. Shielding Method of Printed Circuit Board and Device Mounted Printed Board Using this Method. Japanese Patent 2003078279 A, 14 March 2003.

13. CEDRAT Company Homepage. Available online: http://www.cedrat.com (accessed on 10 October 2011).

14. Paul, C.R. A comparison of the contributions of common mode and differential mode currents in radiated emissions. IEEE Trans. Electromagn. Compat. 1989, 31, 189-193.

15. Vincent, B.; Chadebec, O.; Schanen, J.L. Multipolar expansion sensors for near field characterization. In Proceedings of the 2008 International Symposium on Electromagnetic Compatibility, Hamburg, Germany, September 2008; pp. 1-4.

16. Harrington, R.F. Field Computation by Moment Methods; The Macmillan Company: New York, NY, USA, 1968. 
17. Ruehli, A.E. Inductance calculations in a complex integrated circuit environment. IBM J. Res. Develop. 1972, 16, 470-481.

18. Cangellaris, A.C. Frequency-Domain Finite Element Methods for Electromagnetic Field Simulation: Fundamentals, State of the Art, and Applications to EMI/EMC Analysis. In Proceedings of the 1996 IEEE International Symposium on Electromagnetic Compatibility, Santa Clara, CA, USA, August 1996; pp. 107-116.

19. Aimé, J.; Tran, T.-S.; Clavel, E.; Meunier, G.; Le Floch, Y.; Baudesson, P. Magnetic field computation of a common mode filter using finite element, PEEC methods and their coupling. In Proceedings of the 2008 IEEE International Symposium on Industrial Electronics, Cambridge, UK, 30 June-2 July 2008; pp. 7-12.

20. Simkin, J.; Trowbridge, C.W. On the use of the total scalar potential in the numerical solution of field problems in electromagnetics. Int. J. Numer. Methods Eng. 1979, 14, 423-440.

21. Biro, O.; Preis, K.; Buchgraber, G.; Ticar, I. Voltage driven coils in finite-element formulations using a current vector and a magnetic scalar potential. IEEE Trans. Magn. 2004, 40, 1286-1289.

22. Mayergoyz, I.D.; Bedrosian, G. On calculation of 3-D eddy currents in conducting and magnetic shells. IEEE Trans. Magn. 1995, 31, 1319-1324.

23. Guérin, C.; Meunier, G. 3-D Magnetic Scalar Potential Finite Element Formulation for Conducting Shells Coupled With an External Circuit. IEEE Trans. Magn. 2012, 48, 323-326.

(C) 2013 by the authors; licensee MDPI, Basel, Switzerland. This article is an open access article distributed under the terms and conditions of the Creative Commons Attribution license (http://creativecommons.org/licenses/by/3.0/). 